ITEP-TH-34/98

hep-th/9806150

June, 1998

\title{
Interquark Potential in Schrödinger Representation
}

\author{
K. Zarembo \\ Department of Physics and Astronomy, \\ University of British Columbia, \\ 6224 Agricultural Road, Vancouver, B.C. Canada V6T $1 Z 1$ \\ and \\ Institute of Theoretical and Experimental Physics, \\ B. Cheremushkinskaya 25, 117259 Moscow, Russia \\ E-mail: zarembo@itep.ru/@theory.physics.ubc.ca
}

\begin{abstract}
Static charges are introduced in Yang-Mills theory via coupling to heavy fermions. The states containing static color charges are constructed using integration over gauge transformations. A functional representation for interquark potential is obtained. This representation provides a simple criterion for confinement.
\end{abstract}




\section{Introduction}

The gluon vacuum is expected to determine many properties of the low-energy QCD. According to the most straightforward definition, the vacuum is described by a ground state wave function - the lowest energy solution of the stationary Schrödinger equation. The exact ground state in gluodynamics must be very complicated, but one may hope that a reasonably good approximation can be obtained by means of variational or of some other approximate method if a proper parametrization of the wave functional is used. Such parametrization should respect the symmetries of the Yang-Mills theory, first of all, the gauge invariance. There is a simple way to achieve the invariance of a wave functional - to enforce it by integrating over all gauge transformations [1, 2, 3, 团:

$$
\begin{aligned}
& \Psi[A]=\int[D U] \mathrm{e}^{-S\left[A^{U}\right]}, \\
& A_{i}^{U}=U^{\dagger}\left(A_{i}+\frac{1}{g} \partial_{i}\right) U .
\end{aligned}
$$

Alternative approaches are based on the use of gauge-invariant variables from the very beginning [5] or on fixing of residual gauge symmetry [6].

The representation of a projector on the physical states in a form of the integral over gauge transformations is well known in Yang-Mills theory at finite temperature [7]. In the

present context this method appears to be very convenient for variational calculations, since it allows to make the simplest, Gaussian variational ansatz [1], 3], 目] exactly gauge invariant.

The averaging over the gauge group introduces additional variables, the parameters of gauge transformations, which more or less correspond to longitudinal components of the gluon fields. Introduction of such variables might seem somewhat artificial, since longitudinal degrees of freedom do not propogate and, thus, are unphysical in pure gauge theory. This is true, however, until external color charges are considered. Electric fields of a static charge are longitudinal, so longitudinal components of gauge fields are responsible for color electric forces, which, in their turn, are responsible for confinement. Because averaging over gauge transformations explicitly separates longitudinal degrees of freedom, it provides an appropriate framework for consideration of static color charges and of their interaction [2]. In this paper the averaging over gauges is applied to Yang-Mills theory coupled to heavy fermions, which allows to introduce static charges in a more systematic way.

\section{Coupling to heavy fermions}

The ground state in gluodynamics satisfies Schrödinger equation

$$
H_{\mathrm{YM}} \Psi=E_{\mathrm{vac}} \Psi
$$

where $H_{\mathrm{YM}}$ is Yang-Mills Hamiltonian (in $A_{0}=0$ gauge):

$$
H_{\mathrm{YM}}=\int d^{3} x\left(\frac{1}{2} E_{i}^{A} E_{i}^{A}+\frac{1}{4} F_{i j}^{A} F_{i j}^{A}\right) .
$$


When the wave function is a functional of the gauge potentials, the electric fields act as variational derivatives:

$$
E_{i}^{A}=-i \frac{\delta}{\delta A_{i}^{A}}
$$

We consider $S U(N)$ gauge group with generators $T^{A}$ normalized by $\operatorname{tr} T^{A} T^{B}=-\delta^{A B} / 2$ and sometimes use matrix notations for gauge potentials: $A_{i}=A_{i}^{A} T^{A}$.

The Hamiltonian (2.2) commutes with operators $D_{i} E_{i}^{A}$, and the ground state wave functional is subject to the Gauss' law constraint:

$$
D_{i} E_{i}^{A} \Psi=0 .
$$

This constraint represents in the infinitesimal form the gauge invariance of the ground state $\circledast$ :

$$
\Psi\left[A^{\Omega}\right]=\Psi[A] .
$$

The integral (1.1) can be considered as a general solution of the Gauss' law constraint. Any gauge-invariant state can be represented in this form, the exact ground state in gluodynamics, in particular. Of course, the representation (1.1) is aimed to the use of approximate methods, when some simple form of the action $S[A]$ is employed. In the variational approach of Ref. [1] the action was taken quaratic and diagonal in spatial indices. This variational ansatz was generalized to include longitudinal part of the quadratic form [4] and tree-level condensates [3]. However, since the consideration below is purely kinamatical, we do not restrict ourselves to any particular form of the bare wave functional $\exp (-S[A])$.

In order to introduce color charges, we couple the gauge fields to heavy fermions, so heavy that it is possible to neglect their kinetic term in the Hamiltonian:

$$
H=\int d^{3} x\left(\frac{1}{2} E_{i}^{A} E_{i}^{A}+\frac{1}{4} F_{i j}^{A} F_{i j}^{A}+M \bar{\psi} \psi\right) .
$$

The fermion operators obey anticommutation relations

$$
\left\{\psi_{\alpha}^{a}(x), \psi_{\beta b}^{\dagger}(y)\right\}=\delta_{\alpha \beta} \delta_{b}^{a} \delta(x-y),
$$

where $a, b$ and $\alpha, \beta$ are color and spinor indices, respectively. The fermion fields transform in the fundamental representation of $S U(N)$ :

$$
\psi^{U}=U^{\dagger} \psi, \quad \psi^{\dagger U}=\psi^{\dagger} U
$$

Physical states are gauge invariant and satisfy the Gauss' law constraint:

$$
\left(D_{i} E_{i}^{A}+i \psi^{\dagger} T^{A} \psi\right) \Psi_{\mathrm{phys}}=0 .
$$

Because of the absence of the kinetic term for matter fields, fermion operators obey simple commutation relations with the Hamiltonian. In the standart representation of the Dirac matrices, when $\gamma^{0}$ is diagonal:

$$
\gamma^{0}=\left(\begin{array}{cc}
\mathbf{1} & 0 \\
0 & -\mathbf{1}
\end{array}\right),
$$
[8.

*For simplicity, we disregard peculiarities related to topologically nontrivial gauge transformations, see 
the commutation relations take the form:

$$
\begin{aligned}
& {\left[H, \psi_{\alpha}(x)\right]= \pm M \psi_{\alpha}(x), \quad \begin{cases}+, & \alpha=3,4 \\
-, & \alpha=1,2\end{cases} } \\
& {\left[H, \psi_{\alpha}^{\dagger}(x)\right]= \pm M \psi_{\alpha}^{\dagger}(x), \quad\left\{\begin{array}{ll}
+, & \alpha=1,2 \\
-, & \alpha=3,4
\end{array} .\right.}
\end{aligned}
$$

Therefore, $\psi_{3,4}(x), \psi_{1,2}^{\dagger}(x)$ and $\psi_{1,2}(x), \psi_{3,4}^{\dagger}(x)$ are creation and annihilation operators, respectively. The fermion vacuum is defined by equations

$$
\begin{aligned}
& \psi_{1,2}(x)|0\rangle=0 \\
& \psi_{3,4}^{\dagger}(x)|0\rangle=0 .
\end{aligned}
$$

The fermion vacuum is gauge invariant by itself, and the ground state of the Hamiltonian (2.6) is described by the wave function

$$
\Psi_{\mathrm{vac}}=\Psi[A]|0\rangle=\int[D U] \mathrm{e}^{-S\left[A^{U}\right]}|0\rangle
$$

where $\Psi[A]$ is the vacuum wave functional in gluodynamics - the lowest-energy solution of the Schrödinger equation (2.1).

Excited fermion states are obtained by acting of creation operators on the vacuum. These states are no more gauge invariant, and it is necessary to average them over gauge transfromation in order to project on the physical subspace. The physical states are, consequently, constructed with the help of dressed operators

$$
\begin{aligned}
\psi_{\alpha}^{U a^{\prime}}(y) & =U_{a}^{\dagger a^{\prime}}(y) \psi_{\alpha}^{a}(y), \\
\psi_{\beta b^{\prime}}^{\dagger U}(x) & =\psi_{\beta b}^{\dagger}(x) U_{b^{\prime}}^{b}(x),
\end{aligned}
$$

where $\alpha=3,4$ and $\beta=1,2$ (below spinor indices are omitted for brevity). The most general state of this type,

$$
\begin{aligned}
& \Psi_{a_{1}^{\prime} \ldots a_{n}^{\prime}}^{b_{1}^{\prime} \ldots b_{s}^{\prime}}\left(x_{1}, \ldots, x_{n}, y_{1}, \ldots, y_{s}\right)=\int[D U] \mathrm{e}^{-S\left[A^{U}\right]} \psi_{a_{1}^{\prime}}^{\dagger U}\left(x_{1}\right) \ldots \psi_{a_{n}^{\prime}}^{\dagger U}\left(x_{n}\right) \\
& \psi^{U b_{1}^{\prime}}\left(y_{1}\right) \ldots \psi^{U b_{s}^{\prime}}\left(y_{s}\right)|0\rangle
\end{aligned}
$$

describes $n$ static antiquarks and $s$ quarks located at points $x_{i}, y_{i}$. Dressed operators (2.15) are invariant under local gauge transformations because gauge rotations of the gluon and of the quark fields; $A_{i} \rightarrow A_{i}^{\Omega}, \psi \rightarrow \psi^{\Omega}, \psi^{\dagger} \rightarrow \psi^{\dagger \Omega}$; are compensated by a change of variables $U_{a^{\prime}}^{a} \rightarrow \Omega^{\dagger a}{ }_{b} U_{a^{\prime}}^{b}$ in the integral over the gauge group. These transfromations do not act on indices with prime. To be more presize, this statement is true for transformations with $\lim _{x \rightarrow \infty} \Omega(x)=1$. If $\lim _{x \rightarrow \infty} \Omega(x)=\Omega_{\infty} \neq 1$, the change of variables $U \rightarrow \Omega^{\dagger} U$ violates boundary conditions in the path integral over $U$ and should be accomponied by a global transformation $U \rightarrow U \Omega_{\infty}$. This means that indices with prime are global color ones.

The state (2.16) can be factorized in a product of the gluon and the fermion wave functions. For example, quark-antiquark pair in a color-singlet state is described by the wave function

$$
\Psi_{M}(x, y)=\int[D U] \mathrm{e}^{-S\left[A^{U}\right]} \psi_{a^{\prime}}^{\dagger U}(x) \psi^{U a^{\prime}}(y)|0\rangle=\Psi_{b}^{a}[A ; x, y] \psi_{a}^{\dagger}(x) \psi^{b}(y)|0\rangle,
$$


where

$$
\Psi_{b}^{a}[A ; x, y]=\int[D U] \mathrm{e}^{-S\left[A^{U}\right]} U_{a^{\prime}}^{a}(x) U^{\dagger a^{\prime}}(y) .
$$

The gluon wave function (2.18) transforms in the representation $\bar{N} \otimes N$ of the gauge group:

$$
\Psi_{b}^{a}\left[A^{\Omega} ; x, y\right]=\Omega_{c}^{\dagger a}(x) \Omega_{b}^{d}(y) \Psi_{d}^{c}[A ; x, y]
$$

and satisfies Schrödinger equation for pure Yang-Mills theory.

Again, any state obeying transformation law (2.19) can be represented in the form of an integral (2.18); moreover, the same action $S[A]$ can be used both in the vacuum and in the charged sectors. The integration over gauge transformations projects the bare wave functional $\exp (-S[A])$ on subspaces of states with definite transformation properties, gauge invariant states in the former case and the ones belonging to $\bar{N} \otimes N$ representation in the latter. These subspaces are orthogonal in the full Hilbert space, and it is always possible to find a state which has given projections on each of them. Hence, the action $S[A]$ can be chosen in such a way that the projections of the wave functional $\exp (-S[A])$ on the sectors defined by transfromation laws (2.5) and (2.19) are the lowest-energy eigenstates of the Yang-Mills Hamiltonian in each sector. So, the vacuum, the quark-antiquark and, in fact, any state of type (2.16) can be obtained by averaging of the same bare wave functional over gauge transformations. The above arguments concern exact wave functionals, but the statement that one can use the same action $S[A]$ in different sectors is also true within a variational approach. Suppose that the form of $S[A]$ is fixed up to some parameters, then variational estimates for these parameters will be the same in the vacuum and in the charged sectors [2] because energies of the ground states in these sectors, being proportional to the volume, differ by an amount which is finite in the infinite volume limit and, thus, does alter variational equations.

Another example of the state (2.16) is the one with baryonic quantum numbers:

$$
\begin{aligned}
\Psi_{B}\left(y_{1}, \ldots, y_{N}\right) & =\int[D U] \mathrm{e}^{-S\left[A^{U}\right]} \varepsilon_{a_{1}^{\prime} \ldots a_{N}^{\prime}} \psi^{U a_{1}^{\prime}}\left(y_{1}\right) \ldots \psi^{U a_{N}^{\prime}}\left(y_{N}\right)|0\rangle \\
& =\Psi_{a_{1} \ldots a_{N}}\left[A ; y_{1}, \ldots, y_{N}\right] \psi^{a_{1}}\left(y_{1}\right) \ldots \psi^{a_{N}}\left(y_{N}\right)|0\rangle, \\
\Psi_{a_{1} \ldots a_{N}}\left[A ; y_{1}, \ldots, y_{N}\right] & =\int[D U] \mathrm{e}^{-S\left[A^{U}\right]} \varepsilon_{a_{1}^{\prime} \ldots a_{N}^{\prime}} U_{a_{1}}^{\dagger a_{1}^{\prime}}\left(y_{1}\right) \ldots U_{a_{N}}^{\dagger a_{N}^{\prime}}\left(y_{N}\right) .
\end{aligned}
$$

The state (2.18) has simple physical meaning, it describes color fields of static quarkantiquark pair in the gluon vacuum. In the Abelian theory, Coulomb field of a static charge is created by an operator $\mathrm{e}^{i e V(x)}$ [9], where

$$
V(x)=\int d^{3} y \frac{1}{-\partial^{2}}(x-y) \partial_{i} A_{i}(y)
$$

so dressed electron operators have the form [9]:

$$
\begin{aligned}
\psi_{\alpha}^{(*)}(y) & =\mathrm{e}^{-i e V(y)} \psi_{\alpha}(y), \\
\psi_{\beta}^{(*) \dagger}(x) & =\mathrm{e}^{i e V(x)} \psi_{\beta}^{\dagger}(x) .
\end{aligned}
$$

It is not difficult to reproduce this construction from the representation based on averaging over gauge transformations. In Abelian theory $U=\mathrm{e}^{i e \varphi}, A_{i}^{U}=A_{i}+\partial_{i} \varphi$ and the action $S[A]$ 
should be quadratic; for simplicity we choose the diagonal quadratic form. The integration over gauge transformations in (2.18) is Gaussian and can be done explicitly:

$$
\begin{aligned}
\Psi[A ; x, y]= & \int[d \varphi] \exp \left[-\frac{1}{2} \int d^{3} x d^{3} w\left(A_{i}+\partial_{i} \varphi\right)(z) K(z-w)\left(A_{i}+\partial_{i} \varphi\right)(w)\right. \\
& +i e \varphi(x)-i e \varphi(y)] \\
= & C \mathrm{e}^{i e V(x)} \mathrm{e}^{-i e V(y)} \exp \left(-\frac{1}{2} \int d^{3} z d^{3} w A_{i}^{\perp}(z) K(z-w) A_{i}^{\perp}(w)\right),
\end{aligned}
$$

where $C$ is a field-independent constant and $A_{i}^{\perp}(z)$ denotes transversal part of the gauge potentials:

$$
A_{i}^{\perp}=\left(\delta_{i j}-\frac{\partial_{i} \partial_{j}}{\partial^{2}}\right) A_{j} .
$$

As follows from conformal symmetry, the coefficient function $K$ is equal to $|p|$ in the momentum space. Then the last factor in (2.24) is nothing but the vacuum wave functional for free electro-magnetic field. The first two factors reproduce dressing operators which correspond to a pair of static charges of opposite sign located at $x$ and $y$. More generally, the integration over the Abelian gauge group in (2.16), due to its Gaussian nature, leads to the replacement of $\psi^{U}$ by $\psi^{(*)}$, so dressing (2.15), (2.16) is equivalent to (2.23) in the Abelian theory.

Dressed operators (2.23) possess a number of important properties. They are gaugeinvariant and create eigenstates of the free electro-magnetic Hamiltonian. In QED they also play an important role, because dressed electron and positron operators allow to avoid IR divergencies related to emission of soft photons [10, 11]. Different non-Abelian generalazations of the operators $(2.23)$ were proposed [10, 12]. In contrast with these constructions, dressing (2.16) in non-Abelian case does not have factorized form, the integral over gauge transformations is no more Gaussian, and the operator creating static gauge fields of one particular charge cannot be defined; that is, the form of the wave functional describing this charge depends on the presence of other charges.

\section{Interquark potential}

The energy of the state (2.17) after obvious subtractions determines $q \bar{q}$ interaction potential:

$$
\frac{\left\langle\Psi_{M}|H| \Psi_{M}\right\rangle}{\left\langle\Psi_{M} \mid \Psi_{M}\right\rangle}=2 M+E_{\mathrm{vac}}+V(x-y) .
$$

Matrix elements of gauge-invariant states, like those entering eq. (3.1), are proportional to the volume of the gauge group. The representation (1.1) allows to get rid of this infinite factor easily [1]. For example, the norm of the vacuum state is

$$
\langle\Psi \mid \Psi\rangle=\int[D U]\left[D U^{\prime}\right][d A] \mathrm{e}^{-S\left[A^{U}\right]-S\left[A^{U^{\prime}}\right]}=\mathrm{const} \int[D U][d A] \mathrm{e}^{-S\left[A^{U}\right]-S[A]} .
$$

The norm of the charged state (2.17) is

$$
\left\langle\Psi_{M} \mid \Psi_{M}\right\rangle=\text { const } \int[D U][d A] \operatorname{tr} U(x) U^{\dagger}(y) \mathrm{e}^{-S\left[A^{U}\right]-S[A]} .
$$


Matrix elements of the Hamiltonian correspond to insertion of the operator $R[A]$,

$$
R[A]=\int d^{3} x\left(\frac{1}{2} \frac{\delta^{2} S[A]}{\delta A_{i}^{A} \delta A_{i}^{A}}-\frac{1}{2} \frac{\delta S[A]}{\delta A_{i}^{A}} \frac{\delta S[A]}{\delta A_{i}^{A}}+\frac{1}{4} F_{i j}^{A} F_{i j}^{A}\right),
$$

if variational derivatives in the Hamiltonian act on the right. If they act on the left, $R[A]$ is replaced by $R\left[A^{U}\right]$. It is convenient to introduce a source for the symmetric combination $\left(R\left[A^{U}\right]+R[A]\right) / 2$ :

$$
Z=\int[D U][d A] \mathrm{e}^{-S\left[A^{U}\right]-S[A]+\frac{\lambda}{2} R\left[A^{U}\right]+\frac{\lambda}{2} R[A]},
$$

then matrix elements of the Hamiltonian are obtained by differentiation with respect to $\lambda$. For example, the vacuum energy is given by

$$
E_{\mathrm{vac}}=\left.\frac{\partial}{\partial \lambda} \ln Z\right|_{\lambda=0} .
$$

This quantity is proportional to the volume and contains UV divergent contribution from zero-point oscillations. Regularized energy density can be related to gluon condensate [15]:

$$
E_{\mathrm{vac}}=\frac{\beta\left(\alpha_{s}\right)}{16 \alpha_{s}}\left\langle 0\left|F_{\mu \nu}^{A} F^{A \mu \nu}\right| 0\right\rangle \mathrm{Vol}+\mathrm{UV} \text { divergent terms, }
$$

where $\beta\left(\alpha_{s}\right)$ is a $\beta$-function in Yang-Mills theory.

For $q \bar{q}$ potential we obtain:

$$
V(x-y)=\left.\frac{\partial}{\partial \lambda} \ln \left\langle\operatorname{tr} U(x) U^{\dagger}(y)\right\rangle\right|_{\lambda=0},
$$

where averaging is defined by the partition function (3.5). It is worth explaining how confinement can arise in such representation. The confining potential grows with distance; at the same time, the correlator in (3.8) should decrease at infinity. But its logarithm can increase thus leading to the confining potential. It happens when the integration over the gauge group produces a mass gap:

$$
\left\langle\operatorname{tr} U(x) U^{\dagger}(y)\right\rangle \propto \mathrm{e}^{-m r},
$$

where $r=|x-y| \rightarrow \infty$. Then interquark potential grows linearly with distance:

$$
V(r)=\sigma r+\ldots,
$$

the string tension being equal to the derivative of the mass:

$$
\sigma=-\left.\frac{\partial m}{\partial \lambda}\right|_{\lambda=0} .
$$

Hence, the confinement arises due to generation of a mass gap in the averaging over gauge transformations. More presizely, the confining potential is generated if there is a nonzero linear response of the mass gap to switching of the Hamiltonian. 
Analogously, the energy of quarks in the baryonic state (2.20),

$$
V_{B}\left(y_{1}, \ldots, y_{N}\right)=\frac{\left\langle\Psi_{B}|H| \Psi_{B}\right\rangle}{\left\langle\Psi_{B} \mid \Psi_{B}\right\rangle}-N M-E_{\mathrm{vac}}
$$

can be represented as the derivative of certain correlation function in the statistical system defined by the partition function (3.5):

$$
V_{B}\left(y_{1}, \ldots, y_{N}\right)=\left.\frac{\partial}{\partial \lambda} \ln \left\langle\varepsilon_{a_{1} \ldots a_{N}} \varepsilon^{b_{1} \ldots b_{N}} U_{b_{1}}^{\dagger a_{1}}\left(y_{1}\right) \ldots U_{b_{N}}^{\dagger a_{N}}\left(y_{N}\right)\right\rangle\right|_{\lambda=0}
$$

\section{Discussion}

Examples of calculations of interquark potential starting from representation based on av-

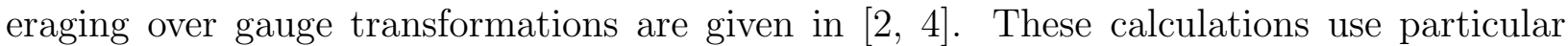
assumptions about the structure of the ground state wave functional in gluodynamics and are by no means derivations from first principles. On the level of kinematical consideration, one can state that the potential is confining if the integration over gauge transformations generates a mass gap with nonzero linear response on switching of the Hamiltonian. The calculations [2, 4] show that generation of a mass gap is rather natural in non-Abelian theory, but quantitative results can be obtained only if the vacuum wave functional is known in some approximation. Perhaps, the simplest Gaussian ansatz averaged over gauge transformations [1, 4, 3] can provide a reasonable variational approximation. The main problem is correct renormalization. The variational method always overestimates the vacuum energy, if non-renormalized divergencies remain, the overestimate is infinite, and variational approximation loses sense [14]. It was argued [1] that Gaussian ansatz can be made compatible with asymptotic freedom; the renormalization properties of Yang-Mills theory in the Schrödinger representation were also discussed in [15] from different points of view.

\section{Acknowledgments}

The author is grateful to I. Kogan, M. Polikarpov and G. Semenoff for discussions. This work was supported by NATO Science Fellowship and, in part, by CRDF grant 96-RP1253, INTAS grant 96-0524, RFFI grant 97-02-17927 and grant 96-15-96455 of the support of scientific schools.

\section{References}

[1] I.I. Kogan and A. Kovner, Phys. Rev. D52 (1995) 3719, hep-th/9408081.

[2] K. Zarembo, Phys. Lett. B421 (1998) 325, hep-th/9710235.

[3] C. Heinemann, C. Martin, D. Vautherin and E. Iancu, hep-th/9802036.

[4] D. Diakonov, hep-th/9805137. 
[5] J. Goldstone and R. Jackiw, Phys. Lett. B74 (1978) 81;

Yu.A. Simonov, Sov. J. Nucl. Phys. 41 (1985) 835 [Yad. Fiz. 41 (1985) 1311];

M. Bauer, D.Z. Freedman and P.E. Haagensen, Nucl. Phys. B428 (1994) 147, hepth/9405028;

P. E. Haagensen and K. Johnson, Nucl. Phys. B439 (1995) 597, hep-th/9408164;

D. Karabali and V.P. Nair, Nucl. Phys. B464 (1996) 135, hep-th/9510157;

P. E. Haagensen, K. Johnson and C. S. Lam, Nucl. Phys. B477 (1996) 273, hepth/9511226.

[6] N.H. Christ and T.D. Lee, Phys. Rev. D22 (1980) 939;

P. van Baal, hep-th/9511119; hep-th/9711070.

[7] A.M. Polyakov, Phys. Lett. B72 (1978) 477; Gauge Fields and Strings (Harwood Academic Publishers, 1987);

L. Susskind, Phys. Rev. D20 (1979) 2610;

B. Svetitsky, Phys. Rep. 132 (1986) 1.

[8] R. Jackiw, Rev. Mod. Phys. 52 (1980) 661.

[9] P.A.M. Dirac, Can. J. Phys. 33 (1955) 650; The Principles of Quantum Mechanics (Oxford, Clarendon Press, 1958).

[10] M. Lavelle and D. McMullan, Phys. Rep. 279 (1997) 1, hep-ph/9509344.

[11] E. Bagan, M. Lavelle and D. McMullan, Phys. Rev. D56 (1997) 3732, hep-th/9602083; D57 (1998) 4521, hep-th/9712080.

[12] P.E. Haagensen and K. Johnson, hep-th/9702204;

M. Lavelle and D. McMullan, hep-th/9805013.

[13] M. Shifman, A. Vainshtein and V. Zakharov, Nucl. Phys. B147 (1979) 385.

[14] G. Tiktopoulos, Phys. Rev. D57 (1998) 6429, hep-th/9705230.

[15] W.E. Brown and I.I. Kogan, hep-th/9705136;

K. Zarembo, hep-th/9803237; hep-ph/9804276. 